\title{
Self-stabilized optoelectronic oscillator using optical feedback on integrated heterodyne source.
}

\author{
A. Thorette $^{1}$, P. Primiani ${ }^{1,2}$, M. Romanelli ${ }^{1}$, M. Alouini ${ }^{1}$, F. Van Dijk ${ }^{2}$, and M. Vallet \\ 1. Institut de Physique de Rennes, UMR Université de Rennes 1-CNRS 6251, Campus de Beaulieu, F-35042 Rennes Cedex \\ 2. III-V Lab, a joint lab of Thales Research and Technology, Nokia Bell Labs France and CEA LETI, F-91767 Palaiseau Cedex
}

Generation of opto-RF signals through heterodyning is now firmly established, the RF frequency being provided by the beat note between two lasers. It offers true single sideband signals, $100 \%$ modulation depth and tunability. However, the use of two independent free-running lasers leads to a large RF linewidth that has to be reduced by means of stabilization schemes. In order to get spectrally pure beat note, we propose to combine passive injection locking of semiconductor lasers with opto-electronic oscillator (OEO) schemes based on optical delaylines. We show that this leads to a semiconductor photonic oscillator, without the need for any RF electronic reference.

(a)

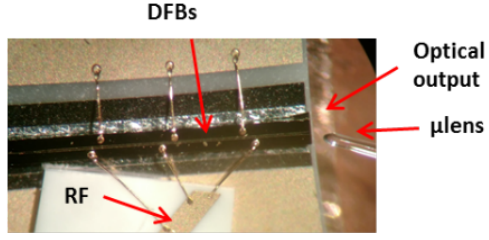

(b)

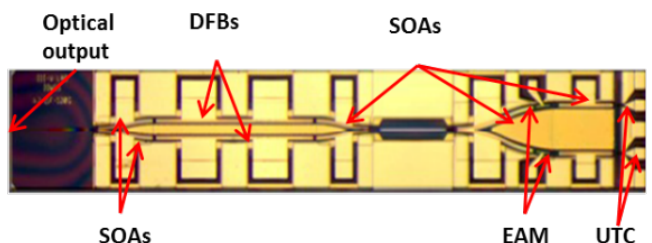

Fig. 1 Monolithic dual DFB lasers used as heterodyne source

The two optical heterodyne sources under consideration consist in specially-designed photonic integrated circuits (PIC), whose optical outputs combine the beams of two monolithic InGaAsP DFB lasers (see Fig. 1). These architectures permit to obtain a small beat note drift as the two lasers share correlated thermal and acoustic fluctuations, and enable one to finely tune the beating frequency up to $100 \mathrm{GHz}$ thanks to the bias current [2,3].
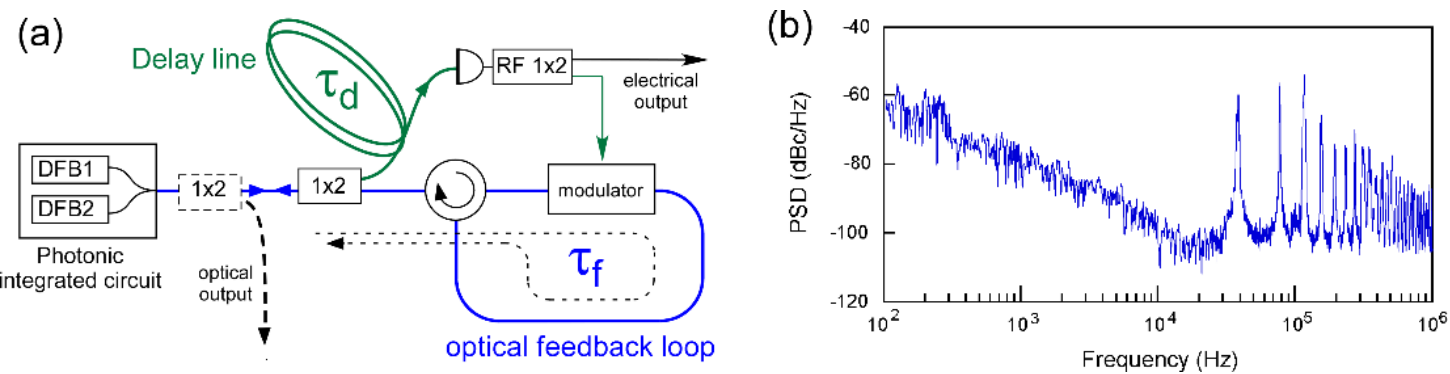

Fig. 2 (a) Generic principle of the optoelectronic oscillator. Blue: frequency-shifted feedback loop. Green: delay line.

(b) Power spectral density of the RF phase. Here, the carrier frequency is set to $12 \mathrm{GHz}$.

The beat note is stabilized by means of a frequency-shifted optical feedback loop (delay $\tau_{\mathrm{f}}$ ) associated with an optical delay line $\tau_{\mathrm{d}}$ (Fig. 2a) [3]. An inloop electro-optic modulator is used to generate sidebands in the feedback optical spectrum at the beat note frequency. Owing to the sensitivity of laser diodes to cross-optical injection, these sidebands, reinjected into the DFBs, induce frequency locking through nonlinear coupling inside the active medium. With the first PIC consisting simply in two monolithic DFBs (Fig. 1a), we find a phase noise level of $65 \mathrm{dBc} / \mathrm{Hz}$, detection limited, at $100 \mathrm{~Hz}$ from a $12 \mathrm{GHz}$ carrier, as shown on Fig. 2b). This was obtained with an optimal $5 \mathrm{~km}$-long delay line. The residual noise peaks are at multiples of $1 / \tau_{\mathrm{d}}$, as expected in OEO architecture. For compactness purpose, we have also tested the stabilization scheme on a more complex PIC, that, in addition to the two DFBs, integrates electro-absorption modulators, SOAs and a UTC photodiode (Fig. 1b). The feedback is provided by reflection on the UTC. This allows drastically reducing the optical loop length. With a short optoelectronic loop of a few meters, we get frequency locking at frequencies ranging from 15 to $20 \mathrm{GHz}$.

Our setup can also be modified by removing the modulator. Direct modulation of the current by the delayed beatnote, combined with an optical feedback, is also expected to provide self-stabilization of the beatnote. Preliminary measurements demonstrate a phase noise level similar to the one shown in Fig. $2 \mathrm{~b}$.

\section{References}

[1] F. Van Dijk et al., "Monolithic dual wavelength DFB lasers for narrow linewidth heterodyne beat-note generation," Proc. Int. Top. Meeting Microw. Photon. MWP/APMP, 73 (2011)

[2] F. Van Dijk et al., "Integrated InP Heterodyne Millimeter Wave Transmitter", IEEE Photon. Technol. Lett. 26, 965 (2014).

[3] M. Vallet, M. Romanelli, G. Loas, F. van Dijk, and M. Alouini, "Self-stabilized optoelectronic oscillator using frequency-shifted feedback and a delay line", IEEE Photon. Technol. Lett. 28, 1088 (2016). 